\title{
Romuald Fonkoua, Aimé Césaire
}

\section{Luigia Pattano}

\section{OpenEdition}

\section{Journals}

\section{Édition électronique}

URL : http://journals.openedition.org/studifrancesi/6467

DOI : 10.4000/studifrancesi.6467

ISSN : 2427-5856

\section{Éditeur}

Rosenberg \& Sellier

\section{Édition imprimée}

Date de publication : 1 novembre 2010

Pagination : 601-602

ISSN : 0039-2944

\section{Référence électronique}

Luigia Pattano, « Romuald Fonkoua, Aimé Césaire », Studi Francesi [En ligne], 162 (LIV | III) | 2010, mis en ligne le 30 novembre 2015, consulté le 11 janvier 2021. URL : http://journals.openedition.org/ studifrancesi/6467 ; DOI : https://doi.org/10.4000/studifrancesi.6467

\section{Ce document a été généré automatiquement le 11 janvier 2021.}

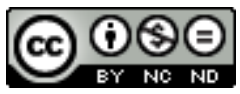

Studi Francesi è distribuita con Licenza Creative Commons Attribuzione - Non commerciale - Non opere derivate 4.0 Internazionale. 


\title{
Romuald Fonkoua, Aimé Césaire
}

\author{
Luigia Pattano
}

\section{RÉFÉRENCE}

Romuald fonkoua, Aimé Césaire, Paris, Perrin, 2010, pp. 395.

1 Deux ans après la mort d'Aimé Césaire (17 avril 2008), Romuald Fonkoua nous livre une biographie dressant un premier bilan intellectuel post mortem du célèbre poète-maire de Fort-de-France, qui vécut en protagoniste une des aventures intellectuelles majeures de son époque - l'affirmation des écrivains et des artistes noirs dans le monde portant, pour lui, le nom de «Négritude» - et qui participa activement, pendant un demi siècle, à la vie politique de son île. Figure aussi fascinante que dérangeante pour la République française, qu'il servit malgré ses ambiguïtés vis-à-vis de son histoire coloniale, qu'il ne cessa de rappeler et de dénoncer tout au long de son existence, ce «nègre fondamental» reste, pour l'auteur de l'ouvrage, «la dernière grande figure d'une génération d'écrivains français et de langue française qui ont allié littérature et politique et ont dû affronter les exigences de l'une et de l'autre, les contradictions soulevées par les pratiques de l'une et l'autre» (p. 358). En fait, le terme de biographie est impropre à désigner ce texte qui n'a rien d'une simple vie d'artiste. Le portrait que Fonkoua propose est en effet celui d'un poète engagé en littérature comme en politique, qui mit sa vie privée entièrement au service de sa vie publique, au prix sans doute de ce bonheur que la paix domestique apporte. Une fois ce texte achevé, le lecteur saura très peu de la famille et des amitiés de Césaire, des rumeurs circulant sur lui, de ses conflits personnels et de ses passions secrètes, de son quotidien et de sa vie intime: ce n'est pas l'homme dans son humaine banalité qui ressort de ce texte, mais l'œuvre d'un grand poète dans sa dimension profondément politique, c'est-à-dire la seule chose qui reste et qui au fond compte en littérature, ce qui en assure la pérennité.

2 Après avoir donné quelques renseignements biographiques concernant l'origine du poète (le milieu où il naquit et grandit, la position sociale de sa famille et ses premières études à la Martinique), Fonkoua s'attarde sur les années parisiennes (1931-1939) de 
Césaire, qui constituèrent un moment fondamental dans sa vie car c'est à Paris qu'il rencontra, par le biais d'«un petit bonhomme noir en blouse grise [...] avec de gros yeux» (p. 44), les deux composantes essentielles de sa vie publique: l'Afrique et la politique. Son «cicéron dans le labyrinthe du savoir et de la montagne SainteGeneviève» (p. 45), Léopold Sédar Senghor, contribua grandement à la prise de conscience du jeune étudiant martiniquais, le mettant en contact avec le réseau d'étudiants noirs qui s'organisait autour de quelques revues, notamment «L'Étudiant noir», et d'une association estudiantine, à la tête de laquelle siègea Césaire. Paris fut donc pour le poète une étape essentielle en ce qu'elle représenta la prise de conscience l'amenant à l'élaboration d'une poétique nègre qui l'associa à Senghor et à Damas, mais fut aussi le lieu de sa formation intellectuelle. Ses années d'études au lycée Louis-LeGrand (1931-1935) et à l'École Normale Supérieure (1935-1939) façonnèrent ses goûts littéraires et son écriture à venir qui allait en effet témoigner d'une passion pour le mot précis, d'un souci d'exactitude lexicale relevant d'une poétique singulière. Paris lui permit enfin de rencontrer Suzanne Roussi, la femme qui l'accompagna dans sa première aventure intellectuelle martiniquaise, la création de la revue «Tropiques», et qui allait rester à ses côtés jusqu'à leur divorce, à la fin des années cinquante.

Précédé par la publication, dans le numéro d'août de la revue «Volonté», du Cahier d'un retour au pays natal, dont nous est donnée la suggestive génèse, le retour au pays en 1939 fut pour le couple Césaire le début d'un intense travail d'animation culturelle à travers la formation des futurs intellectuels de l'île (l'enseignement secondaire) et surtout par l'entreprise de «Tropiques». CEuvre triomphante d'Aimé et de Suzanne, la revue qui stupéfia Breton fut le lieu d'une révolution poétique sans précédent pour la Martinique, de l'affirmation de la Négritude et de la prise de conscience de l'importance de l'Afrique dans la composition de la culture antillaise. Cette aventure prit fin lors de l'entrée du poète en politique: le 27 mai 1945, à l'age de 32 ans, Césaire fut élu maire de Fort-de-France. L'activité politique de Césaire, commencée au sein du Parti communiste, allait durer jusqu'en 2001, absorbant complètement sa vie privée qui se partagea entre Paris, où il exerçait ses fonctions de député à l'Assemblée Nationale et où sa femme et ses enfants s'installèrent, et Fort-de-France. L'action politique se doubla, chez Césaire, d'une activité poétique et culturelle importante. À Paris, il participa aux principaux débats lancés dans les revues qui animaient la vie intellectuelle de l'époque. C'est justement dans une revue, «Chemins du monde», qu'il publia, sous le titre de L'impossible contact, la première version du Discours sur le colonialisme, qui allait connaître de successives et plus célèbres réécritures. Au débat sur le colonialisme suivit, en 1955 , le débat sur la poésie qui l'amena à esquisser les traits d'une ars poetica nègre, en réponse aux déclarations d'Aragon Sur la poésie nationale parues dans «Lettres françaises». Césaire était désormais un personnage très influent dans le panorama culturel français, comme le montre la place qu'il eut au sein du premier Congrès des écrivains et artistes noirs qui se déroula l'année suivante à Paris. Sa contribution au Congrès, intitulée "Culture et colonisation», fut celle qui enflamma davantage le public. Il y soulignait l'importance de l'imbrication entre le politique et le culturel, et invitait à concevoir un art politique dégagé de toute idéologie et contribuant à la décolonisation. La contradiction entre les propos du poète martiniquais et sa position dans le PCF, qui était celle de l'intellectuel organique, était en cette circonstance évidente, comme le souligne fort justement Fonkoua. Mais Césaire allait bientôt la résoudre en démissionnant bruyamment du parti, le 24 octobre de la même année. Cette rupture qui lui coûta l'isolement (à cause des pratiques de 
dénigrement mises en œuvre par les chefs du parti vis-à-vis de ses traitres) eut deux conséquences importantes dans la vie du poète-maire: la fondation d'un nouveau parti (le Parti progressiste martiniquais) et l'abandon progressif de la poésie en faveur du théâtre. Ce fut toujours en 1956 que la revue «Présence Africaine» publia une première version de Et les chiens se taisaient. Césaire, qui avait découvert le théâtre à Paris dans l'entre-deux-guerre se passionnant pour les tragédies de Giraudoux mises en scène par Jouvet, allait non seulement inaugurer le théâtre aux Antilles, mais allait également établir les fondements d'une tragédie antillaise. Aux Antilles, où aucun mythe n'existe, la tragédie devait endosser, pour Césaire, la fondation du mythe. Par ce biais, elle devait nommer et mettre enfin en valeur les Martiniquais et leur histoire méconnue, voire occultée, et contribuer ainsi à leur prise de conscience. Cette conception de la tragédie allait être développée dans le triptyque théâtral dont se compose sa dernière production qui s'inscrit dans le théâtre militant: La tragédie du roi Christophe, Une saison au Congo et Une tempête. Les trois volets du triptyque, qui se déroulent respectivement en Haïti, au Congo et aux États-Unis et qui ont pour objet la situation contemporaine des noirs dans ces trois parties du monde, témoignent d'une idée du théâtre politique, dont le but serait, d'après Césaire lui-même, de «prendre en charge le passé, éclairer le présent, débusquer l'avenir, bref, aider à achever et à conduire à sa vraie naissance l'archipel inachevé» (p. 344).

4 Sur la dernière pièce de Césaire se ferme le livre. Ayant pris le parti de rendre compte de l'aventure poétique et intellectuelle du poète et dramaturge, les trente dernières années de son existence ne sont pas considérées par Fonkoua qui nous laisse pourtant deux instantanés presque pathétiques de Césaire à la fin de sa trajectoire existentielle. Ces images de la fin renvoyant aux deux dernières entrevues avec le poète-maire à la Martinique (la première remontant à 2006, la seconde à février 2008) nous sont présentées, en fait, au tout début du texte, dans le «Prologue». Il s'agit des seules images du Césaire en chair et en os que l'auteur nous offre, d'une petite parenthèse dans ce texte qui interroge l'œuvre sans considérer l'homme et ses relations humaines. Des instantanés significatifs car ils révèlent, pour Fonkoua, deux traits saillants du caractère de Césaire: la force et la simplicité. Si le premier ne nécessite d'aucune explication car il se trouve dans chaque acte d'énonciation de l'intellectuel et du politicien, le second est par contre le seul jugement concernant la sphère privée que Fonkoua se concède. La seule exception dans un ouvrage qui situe bien l'œuvre du Césaire poète et dramaturge dans son temps car, et Fonkoua le prouve de façon convaincante, il n'est pas essentiel de recourir aux données personnelles, aux souvenirs et aux témoignages des proches pour l'analyse littéraire de l'œuvre. 